\title{
The ancient function of RB-E2F Pathway: insights from its evolutionary history
}

\author{
Lihuan $\mathrm{CaO}^{\dagger}$, Bo Peng ${ }^{\dagger}$, Lei Yao, Xinming Zhang, Kuan Sun, Xianmei Yang, Long Yu ${ }^{*}$
}

\begin{abstract}
Background: The RB-E2F pathway is conserved in most eukaryotic lineages, including animals and plants. E2F and RB family proteins perform crucial functions in cycle controlling, differentiation, development and apoptosis. However, there are two kinds of E2Fs (repressive E2Fs and active E2Fs) and three RB family members in human. Till now, the detail evolutionary history of these protein families and how RB-E2F pathway evolved in different organisms remain poorly explored.
\end{abstract}

Results: We performed a comprehensive evolutionary analysis of E2F, RB and DP (dimerization partners of E2Fs) protein family in representative eukaryotic organisms. Several interesting facts were revealed. First, orthologues of $\mathrm{RB}, \mathrm{E} 2 \mathrm{~F}$, and DP family are present in several representative unicellular organisms and all multicellular organisms we checked. Second, ancestral E2F, RB genes duplicated before placozoans and bilaterians diverged, thus E2F family was divided into E2F4/5 subgroup (including repressive E2Fs: E2F4 and E2F5) and E2F1/2/3 subgroup (including active E2Fs: E2F1, E2F2 and E2F3), RB family was divided into RB1 subgroup (including RB1) and RBL subgroup (including RBL1 and RBL2). Third, E2F4 and E2F5 share more sequence similarity with the predicted E2F ancestral sequence than E2F1, E2F2 and E2F3; E2F4 and E2F5 also possess lower evolutionary rates and higher purification selection pressures than E2F1, E2F2 and E2F3. Fourth, for RB family, the RBL subgroup proteins possess lower evolutionary rates and higher purification selection pressures compared with RB subgroup proteins in vertebrates,

Conclusions: Protein evolutionary rates and purification selection pressures are usually linked with protein functions. We speculated that function conducted by E2F4/5 subgroup and RBL subgroup proteins might mainly represent the ancient function of RB-E2F pathway, and the E2F1/2/3 subgroup proteins and RB1 protein might contribute more to functional diversification in RB-E2F pathway. Our results will enhance the current understanding of RB-E2F pathway and will also be useful to further functional studies in human and other model organisms.

Reviewers: This article was reviewed by Dr. Pierre Pontarotti, Dr. Arcady Mushegian and Dr. Zhenguo Lin (nominated by Dr. Neil Smalheiser).

\section{Background}

The RB-E2F pathway is crucial for regulating cell cycle progression and tumorigenesis [1]. Proteins that are related to the retinoblastoma tumor suppressor RB and the E2F transcription factor are conserved in most eukaryotic lineages, including animals and plants [2].

The retinoblastoma susceptibility gene was the first tumor suppressor gene to be identified. RB-family members are generally believed to function through their effects on the transcription of genes regulated by the

\footnotetext{
* Correspondence: longyu@fudan.edu.cn

† Contributed equally

State Key Laboratory of Genetic Engineering, Institute of Genetics, School of Life Sciences, Fudan University, Shanghai 200433, PR China
}

E2F proteins. In human, RB1 (pRb), RBL1 (p107), and RBL2 (p130) constitute a small RB family [1,2].

E2F proteins, which share a conserved DNA binding domain, can bind to overlapping sets of target promoters. In human, there are eight E2F genes (E2F1, E2F2, E2F3, E2F4, E2F5, E2F6, E2F7, and E2F8) [2-4]. E2F1-6 all possess one E2F-TDP domain, and E2F1, E2F2, and E2F3 are generally considered as the 'active E2Fs' on the basis of their ability to potently activate transcription. E2F4 and E2F5 are named as the 'repressive E2Fs', which bind their targets coincident with their repression in G0/G1, and only modestly activate transcription [3,4]. E2F1-5, combined with DP family proteins, can interact with the 'pocket protein' family protein (RB1, RBL1, and

\section{C) Biomed Central}


RBL2). DP family proteins, which contain one E2F-TDP domain and one DP domain, are dimerization partners of E2Fs. E2F6, also possessing one E2F-TDP domain but no RB binding domain, can not bind to RB family proteins [5].

E2F7 and E2F8 own two E2F-TDP domains, and can bind to DNA in the absence of interaction with a DP subunit. However, they lack sequences required for RB family protein binding [2-4].

We will use E2F1-6 family to refer to classic E2F proteins (E2F1, E2F2, E2F3, E2F4, E2F5 and E2F6), and $\mathrm{E} 2 \mathrm{~F} 7 / 8$ family to refer to E2F7 and E2F8 proteins in this manuscript.

During past two decades, a large number of studies, mainly conducted on flies, worms and vertebrates $[2,6,7]$, have characterized the molecular properties and functions of RB-E2F pathway. It has been revealed that $\mathrm{RB}$ family proteins and E2F family proteins function in a wide range of biological processes, including DNA replication, mitosis, mitotic checkpoint, DNA-damage checkpoints, DNA repair, differentiation, development and apoptosis $[2,6,7]$. The functional conservation of RB-E2F pathway in different organisms (human, mouse, worm and fly) were found and reviewed [2]. Although, distributions of transcription factors including E2F, RB, DP proteins were reported in eukaryotic lineages recently [8], the detail evolutionary history of E2F family has not been explored. The evolutionary history of RB family had been investigated previously, but usually only with limited organisms $[9,10]$.

Taking the advantage that more and more genomes had been completely sequenced, we probed the evolutionary history of RB-E2F genes in eukaryotic lineages. Totally, 21 representative eukaryotic organisms were selected for E2F, RB, and DP proteins identification. There are 16 organisms from metazoan (Homo sapiens; Canis familiaris; Bos Taurus; Mus musculus; Rattus norvegicus; Gallus gallus; Xenopus tropicalis; Danio rerio; Tetraodon nigroviridis; Strongylocentrotus purpuratus; Branchiostoma floridae; Ciona intestinalis; Caenorhabditis elegans; Drosophila melanogaster; Nematostella vectensis; and Trichoplax adhaerens), one choanoflagellate (Monosiga brevicollis), two plants (Arabidopsis thaliana; Oryza sativa), one social amoebae (Dictyostelium discoideum AX4), and one green algae(Ostreococcus tauri).

Previously, it was thought that RB-E2F pathway was missed in fungi [2], and it was true for yeast (such as $S$. pombe and S. cerevisiae). However, we found that some fungi own E2F family and DP family proteins, for example: Encephalitozoon cuniculi, belonging to microsporidia which are once thought to be protists but now known to be fungi [11], owns one E2F1-6 family protein (gi (|19074054), and one DP family protein (gi| 19074276). However, no RB family protein is found in
E. cuniculi and other Fungi, and so RB-E2F pathway might be not complete in fungi, we did not cover fungi proteins in detail analysis.

It is worth to mention that several reasons made some representative model organisms to be selected. The placozoan T. adhaerens, represents a primitive metazoan form, and is a basal eumetazoan lineage that diverged before the separation of cnidarians and bilaterians [12]. The sea anemone $N$. vectensis is a non-bilaterian animal, a member of the phylum Cnidaria [13]. The unicellular choanoflagellate (M. brevicollis) is the closest relatives of metazoans, which represents a distinct lineage that evolved before the origin and diversification of metazoans [14]. D. discoideum AX4, a soil amoeba, branched from the lineage that ultimately led to the metazoa before yeast but after plants, the social amoebae are exceptional in their ability to alternate between unicellular and multicellular forms [15]. O. tauri is a genus of unicellular coccoid or spherically shaped green alga, and is the smallest known free-living eukaryote [16].

In this study, we explored particularly, (1) the distribution of RB, E2F genes in eukaryotic lineages; (2) the details about gene duplication events and evolutionary history of RB, E2F genes in metazoa; (3) different evolution rates and selection pressures in subgroup proteins of RB, E2F family; (4) the function insights from the evolutionary history of RB-E2F pathway.

\section{Methods}

\section{Protein Sequence identification}

Using Human E2F4, DP1, E2F7, and RB protein as a query, we performed PSI-Blast searches (E-value less than e- 5 as cut-off) at the National Center for Biotechnology Information (NCBI) Web site http://www.ncbi. nlm.nih.gov/ to screen the non-redundant protein database from 21 organisms [17]. All the new results were used as queries to carry out a second round of BLAST search, until no new sequence was found. BLASTP search was also performed in Ensembl database http:// www.ensembl.org/ for above organisms. In addition, TBLASTN searches were also carried out at (NCBI) Web site. The collected protein sequences were then analyzed by SMART [18] and Pfam [19] for domain architecture.

As E2F1-6 family, E2F7/8 family, and DP family share some sequence similarity, all of them own the E2F-TDP domain, we use below criterions to classify them: all E2F1-6 members only have one E2F-TDP domains, and share more sequence similarity to human E2F4 than to human E2F7 or DP1; All E2F7/8 members have two E2F-TDP domains, and share more sequence similarity to human E2F7 than to human E2F4 or DP1; all DP family members have one E2F-TDP domain and one DP domain, and share more sequence similarity to human 
DP1 than to human E2F4 or E2F7. Some retrieved sequences were discarded on the basis of the following criteria: (1) partial sequences or sequences resulting from frameshifts in the underlying mRNA as a result of cloning artifacts or possible aberrant alternative splicing; (2) Protein sequences which did not contain almost the entire E2F-TDP domain or RB domain (3) duplicated database submissions of the same sequence; and alternatively spliced isoforms.

\section{Protein name used in this study}

For proteins from $H$. sapiens, M. musculus, C. elegans, D. melanogaster, A. thaliana, and O. sativa, we named them by using their symbols in genbank. For proteins from other organisms, which are unnamed in genbank, we named them on the basis of their evolutionary relationship to human proteins. For all the proteins, we also added their organisms as suffix. Abbreviations of organism names can be found after the conclusions section.

\section{Phylogenetic analysis}

Multiple alignments were performed by MUSCLE 3.6 [20] and Clustal X [21] with the default settings. Maximum likelihood (ML) trees were constructed by using PHYML V.2.4 [22], with 500 bootstrap resamplings and JTT setting, and Gamma parameter values were estimated from the data set using the Tree-Puzzle program [23]. Bayesian inference (BI) was performed using the MRBAYES (version 3) package [24], with a mixture of protein evolution models, fixed rate, 100,000 generations, sampling every 100th generation and discarding initial $25 \%$ trees.

\section{Prediction of Ancestral Protein Sequences}

Gapped Ancestral Sequence Prediction program (GASP) [25] was used to predict ancestral sequences from phylogenetic trees and the corresponding multiple sequence alignments.

\section{Computing Protein distances and selection pressures}

Pairwise distances between proteins were calculated in MEGA 4.0 [26] with the amino acid Poisson correction model, uniform rates among sites and lineages, pairwise deletion. The protein-coding DNA sequences were collected from genbank http://www.ncbi.nlm.nih.gov/ and Ensembl database http://www.ensembl.org/. The protein-coding DNA sequences were aligned based on their protein alignments at the web server http://www.bork. embl.de/pal2nal/[27]. The codeml program in PAML (3.0) [28] was used for estimating synonymous and nonsynonymous substitution rates $(K a / K s, K a, K s)$ in pairwise comparisons of protein-coding DNA sequences with the Nei-Gojobori method [29]. For group average pairwise distance value comparisons, all pairwise distance values for all protein sequences in each subgroup were computed. Then average values of group pairwise distances were calculated. Pair Student's T Test was used as statistical evaluation. For group average $\mathrm{Ka} /$ $k s$ value comparisons, all $\mathrm{Ka} / \mathrm{ks}$ values for all proteincoding DNA sequences in each subgroup were first computed for further study.

\section{Results}

The origination and distribution of E2F1-6, E2F7-8, RB, and DP family proteins in Eukaryotes

Based on protein similarity searching and domain associations checking (Detail in material and methods section), E2F1-6, E2F7/8, RB, DP family proteins were identified in representative organisms. At last, 70 E2F1-6 family proteins, 25 E2F7/8 family proteins, 34 DP family proteins, and $41 \mathrm{RB}$ family sequences were identified in 21 eukaryotic organisms (Figure 1). The accession numbers and sequences of all these proteins are listed in additional File 1.

We found that unicellular organisms, $D$. discoideum AX4, O. tauri, and M. brevicollis contain the representative orthologues of E2F1-6, DP, and RB family (figure 1). Based on the phylogenetic relationship among fungi and other organisms, the absence of RB and E2F genes in some fungi (such as S. pombe and S. cerevisiae) might due to gene losses in evolution. And as mentioned in the introduction section, fungi E. cuniculi, own E2F family protein (gi:19074054) and DP family proteins (gi:19074276), but no RB family protein.

For the multicellular organisms, we found that all multicellular lineages possess orthologues of E2F1-6, $\mathrm{DP}$, and RB family. E2F7/8 family genes were present in protist $M$. brevicollis, animals, and plants. So E2F7/ 8 family originated before the separation of protists, animals, and plants. However, orthologues of E2F7/8 were not found in D. melanogaster, C. elegans, B. floridae, and S. purpuratus. We speculated that E2F7/8 family was not as indispensable as E2F1-6, RB, and DP family.

\section{Detailed Evolutionary relationship of the E2F, RB, and DP} family in metazoa

Phylogenetic analysis for E2F, RB and DP proteins from representative eukaryotic organisms were carried out (results could be found in additional File 2). However, the higher sequence variation from distantly related eukaryotic organisms disrupted the global phylogenetic results, and made global phylogenetic trees not very robust and reliable. As our main interest was on the evolution of RB-E2F pathway in metazoa, the phylogenetic analyses on E2F, RB, and DP family in metazoan organisms were performed, and the results were discussed in detail. 


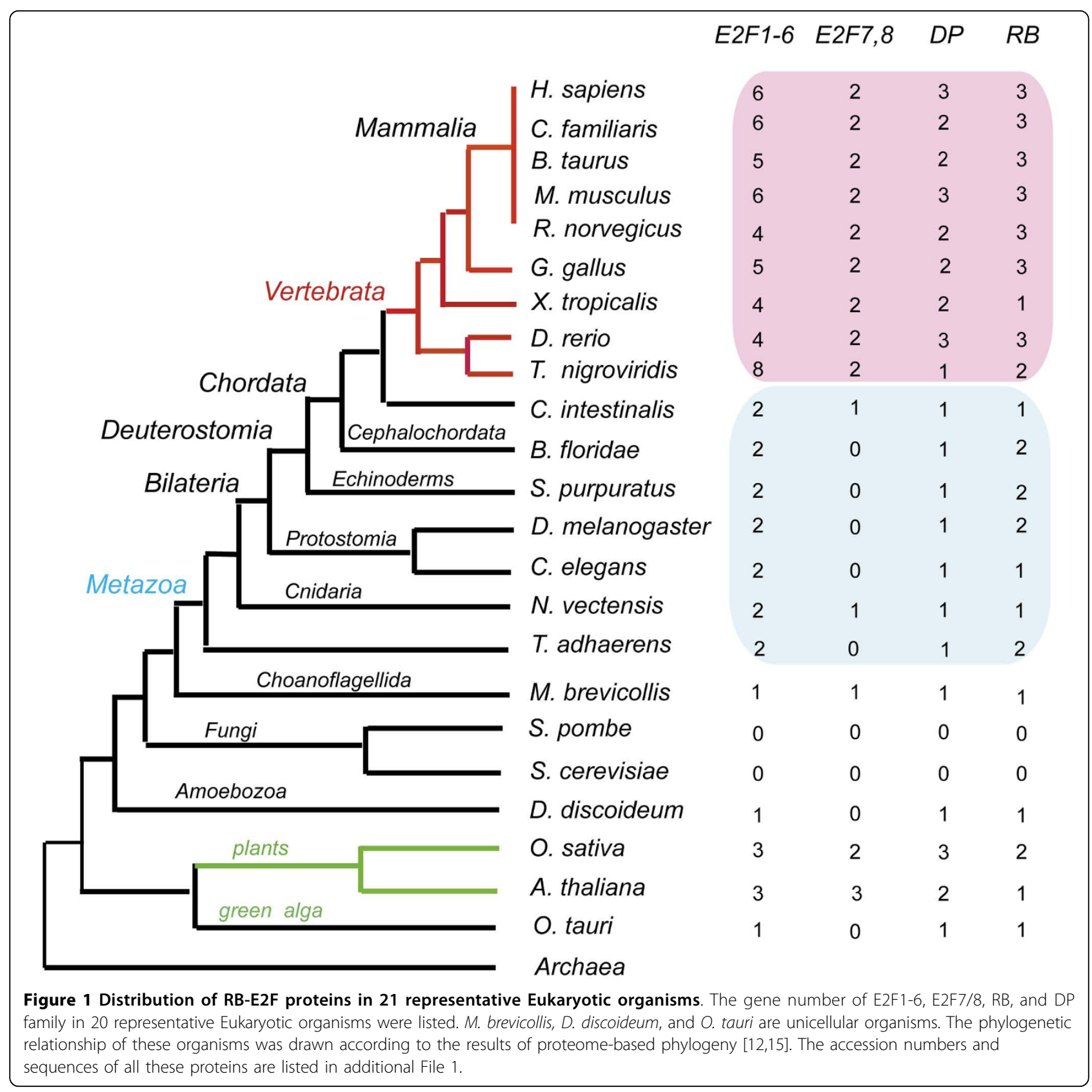

Evolution of E2F1-6 family, E2F7/8 family proteins in metazoa

There are 61 E2F1-6 family proteins identified in 16 metazoan genomes (Figure 1). Both maximum likelihood method (ML) and Bayesian Inference method (BI) were employed for phylogenetic analyses, E2F-Mb (gi:167523471), the E2F protein from M. brevicollis, was used as an outgroup. Both methods produced nearly identical topologies (Figure 2).

In our analysis, E2F1-6 family proteins were divided into E2F4/5 subgroup and E2F1/2/3 subgroup with high statistical supports (Figure 2A). In vertebrates, the E2F1/
2/3 subgroup could be again divided into two classes: E2F1/2/3-vert subgroup and E2F6 subgroup (Figure 2). The genbank accession numbers of proteins in the Figure 2 could be found in additional File 1. E2F1-6 family proteins in Figure 2A were also summarized in Table 1.

It was found that the placozoan, $T$. adhaerens, owns bone fide member E2F1/2/3-Ta (gi:196010483) in E2F1/ 2/3 subgroup, and E2F4/5-Ta (gi:196012606) in E2F4/5 subgroup. Non-bilaterian animal sea anemone (N. vectensis), bilaterian invertebrates S. purpuratus, B. floridae, and $C$. intestinalis all possess their representative genes in E2F1/2/3 subgroup and E2F4/5 subgroup (Figure 2, 


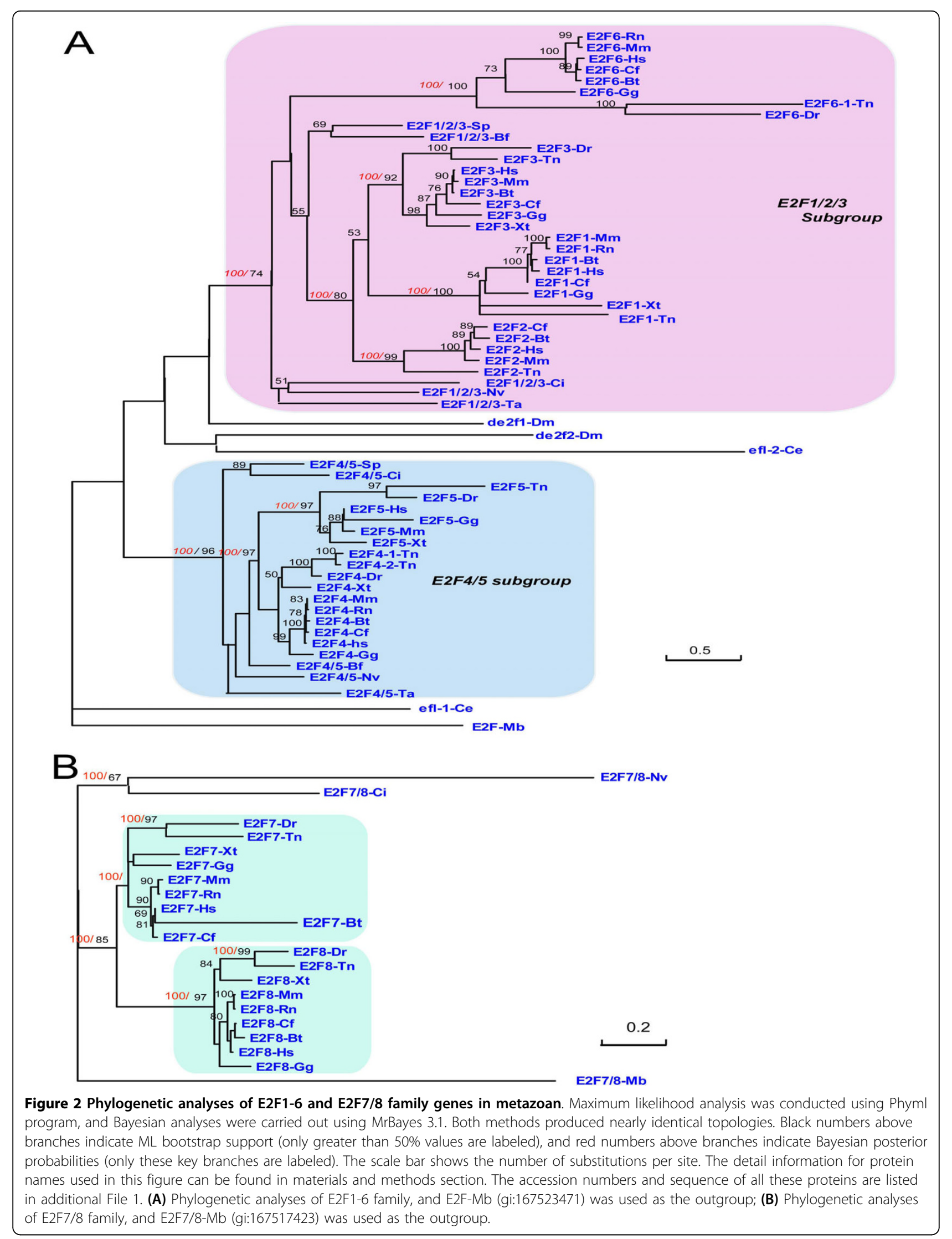


Table 1 Summary of the distribution and sub-grouping of E2F1-6 Family proteins in different organisms

\begin{tabular}{|c|c|c|c|c|c|c|}
\hline & \multicolumn{6}{|c|}{ E2f1-6 family } \\
\hline \multirow[t]{2}{*}{$\begin{array}{l}\text { M } \\
\text { brevicollis }\end{array}$} & \multicolumn{6}{|l|}{ E2F-Mb (gi|167523471) } \\
\hline & \multicolumn{4}{|c|}{ E2F1/2/3 subgroup } & \multicolumn{2}{|l|}{ E2F4/5 subgroup } \\
\hline $\begin{array}{l}\text { T. } \\
\text { adhaerens }\end{array}$ & \multicolumn{4}{|c|}{ E2F1/2/3-Ta (gi|196010483) } & \multicolumn{2}{|l|}{ E2F4/5-Ta (gi|196012606) } \\
\hline $\begin{array}{l}N . \\
\text { vectensis }\end{array}$ & \multicolumn{4}{|c|}{ E2F1/2/3-Nv (gi|156371340) } & \multicolumn{2}{|l|}{ E2F4/5-Nv (gi|156368461) } \\
\hline C. elegans & & & & & \multicolumn{2}{|l|}{ EFL-1-Ce (gi|17559226) } \\
\hline $\begin{array}{l}\text { D.me } \\
\text { lanogaster }\end{array}$ & \multicolumn{4}{|l|}{ E2F1-Dm (gi|24648770) } & \multicolumn{2}{|l|}{ E2F2-Dm (gi|17137542) } \\
\hline $\begin{array}{l}\text { S. } \\
\text { purpuratus }\end{array}$ & & & & & \multicolumn{2}{|l|}{ E2F4/5-Sp (gi|115696783) } \\
\hline $\begin{array}{l}\text { C. } \\
\text { intestinalis }\end{array}$ & \multicolumn{4}{|c|}{ E2F1/2/3-Ci (gi|118343729) } & \multicolumn{2}{|l|}{ E2F4/5-Ci (gi|118343737) } \\
\hline \multirow[t]{2}{*}{ B. floridae } & \multicolumn{4}{|c|}{ E2F1/2/3-Bf (gi|260790430) } & \multicolumn{2}{|l|}{ E2F4/5-Bf (gi|260798626) } \\
\hline & E2F1 & E2F2 & E2F3 & E2F6 & E2F4 & E2F5 \\
\hline $\begin{array}{l}\text { T. } \\
\text { nigroviridis }\end{array}$ & ENSTNIT00000007876 & ENSTNIT00000020634 & ENSTNIT00000012687 & $\begin{array}{l}\text { ENSTNIT00000008935 } \\
\text { ENSTNIT00000013829 }\end{array}$ & $\begin{array}{l}\text { ENSTNIT00000002389 } \\
\text { ENSTNIT00000002758 }\end{array}$ & ENSTNIT00000004791 \\
\hline D. rerio & & & gi|220673319 & gi|71892405 & gi|47087407 & gi|68533607 \\
\hline $\begin{array}{l}X . \\
\text { tropicalis }\end{array}$ & ENSXETT00000046332 & & gi|58331835 & & gi|167560905 & gi|188528909 \\
\hline G. gallus & gi|45382583 & & gi|118086362 & ENSGALT00000031596 & gi|118096144 & gi|71896455 \\
\hline $\begin{array}{l}R \text {. } \\
\text { norvegicus }\end{array}$ & gi|189217865 & & gi|212549627 & gi|109479090 & ENSRNOT00000021145 & \\
\hline $\begin{array}{l}\text { M. } \\
\text { musculus }\end{array}$ & gi|6681243 & gi|29244208 & gi|83523736 & gi|237681138 & gi|22507329 & gi|31982405 \\
\hline B. taurus & gi|194672360 & gil76611569 & gi|76663083 & gi|116003911 & gi|115497534 & \\
\hline $\begin{array}{l}\text { C. } \\
\text { familiaris }\end{array}$ & gil73992245 & ENSCAFT00000021059 & gi|74004128 & gi|73980432 & gi|73957515 & gi|73999542 \\
\hline H. sapiens & gi|12669911 & gi|4758226 & gi|4503433 & gi|109637795 & gi|12669915 & gi|134142811 \\
\hline
\end{tabular}

According to results from Figure 2A, E2F1-6 family proteins (accession numbers from genbank or Ensembl database) identified in M. brevicollis and 16 metazoan organisms were listed. The detail explains could be found in main text.

Table 1). This indicated that the first gene duplication for E2F1-6 family happened before placozoans and bilaterians separation.

In vertebrates, the number of E2F1-6 family member dramatically increased. All human E2F1-6 family proteins could found their direct orthologues in $T$. nigroviridis (Figure 2, Table 1), indicating all human E2F1-6 family proteins emerged in early vertebrates, and gene duplications in E2F4/5 subgroup and E2F1/2/3 subgroup happened before tetrapoda and teleostei divergence (Figure 2, Table 1). In detail, our results demonstrated that human E2F1, E2F2, E2F3 and E2F6 emerged by gene duplications of the common E2F1/2/3 subgroup ancestor. And E2F1/2/3 subgroup members from invertebrates $T$. adhaerens, S. purpuratus, B. floridae, and $C$. intestinalis, which we named them as E2F1/2/3-Ta, E2F1/2/3-Sp, E2F1/2/3-Ci, and E2F1/2/3-Bf, should be recognized as the co-orthologues of human E2F1, E2F2, E2F3 and E2F6 genes. Human E2F4 and E2F5 emerged by gene duplications of the common E2F4/5 subgroup ancestor. And E2F4/5 subgroup members from invertebrates T. adhaerens, $N$. vectensis, S. purpuratus, B. floridae, and $C$. intestinalis, which we named them as E2F4/ 5-Ta, E2F4/5-Nv, E2F4/5-Sp, E2F4/5-Bf, E2F4/5-Ci, should be recognized as the co-orthologues of human E2F4 and E2F5 genes. It needs to be mentioned that though E2F6 emerged together with E2F1, E2F2 and E2F3 from E2F1/2/3 subgroup ancestor, it lost the RB binding domain and did not function in classical RBE2F pathway [2], and we will not discuss it later.

Species-specific gene duplications and gene losses were also found in E2F1-6 family in vertebrates. $H$. sapiens, $C$. familiaris, M. musculus all own 6 members of E2F1-6 family, $T$. nigroviridis has 8 members of E2F1-6 family, with two E2F4 (E2F4-1-Tn(ENSTNIT00000002389), E2F4-2-Tn (ENSTNIT00000002758)) and two E2F6 (E2F6-1-Tn(ENSTNIT00000008935), E2F6-2-Tn (ENSTNIT00000013829)). There are 5 members of 
E2F1-6 family in B. Taurus, with E2F5 orthologue absent; 4 members in $R$. norvegicus, with E2F2 and E2F5 orthologues absent; 5 members in G. gallus, with E2F2 orthologue absent; 4 members in $X$. tropicalis, with E2F2, E2F6 orthologues absent; 4 members in D. rerio, with E2F1 and E2F orthologues absent (Figure 2, Table 1).

It is a little surprise that so many gene losses happened in the E2F1-6 family in vertebrates. However, we found that all vertebrates have at least one member from E2F1/2/3 subgroup, and also one member from E2F4/5 subgroup (Figure 2, Table 1).

The ancestral E2F gene duplication happened before placozoans and bilateria separation. Therefore, bilateria D. melanogaster and C. elegans should theoretically have representative orthologues of E2F4/5 subgroup and E2F1/2/3 subgroup. However, in our analysis, dE2F1Dm, dE2f2-Dm, efl-1-Ce, and efl2-Ce all failed to be clustered into E2F1/2/3 subgroup or E2F4/5 subgroup, possibly due to their high sequence divergence.

In the further analysis (such as Blast), we found that dE2F2-Dm, EFL-1-Ce share more sequence similarity with mammalian E2F4, E2F5 than with E2F1, E2F2, E2F3. In contrast, dE2F1-Dm shares more sequence similarity with E2F1, E2F2, E2F3 than with E2F4, E2F5. In addition, conversation of exon-intron structure for dE2F2-Dm, EFL-1-Ce, human E2F4, and E2F5 gene (Figure 3, additional file 3) was also found. We speculated that dE2F2-Dm and EFL-1-Ce belong to E2F4/5 subgroup, while dE2F1-Dm belong to E2F1/2/3 subgroup. And this classification was also consistent with results of previous functional studies which indicated that EFL-1-Ce and E2F2-Dm usually functioned as repressive E2Fs, while E2F1-Dm functioned as active E2Fs [2]. For EFL-2-Ce, as no function was reported and its sequence is highly diverged, we have no cues whether EFL-2-Ce is a product of species-specific duplication from EFL-1-Ce or a function lost gene which early derived from E2F1/2/3 subgroup. And we will not discuss it later. In anyway, the representative functional gene from E2F1/2/3 subgroup was absent in worm.

For E2F7/8 family (Figure 2), as mentioned in previous section, E2F7/8 family originated in early eukaryotes. However, orthologues of E2F7/8 subgroup were absent in S. purpuratus, B. floridae, C. elegans, and D. melanogaster (Figure 2B, Table 2). For E2F7/8 family, the first gene duplication happened before tetrapoda and teleostei divergence. By the way, protein E2F8-Xt (ENSXETG 00000004436), which did not have the first E2F-TDP domain, and only have the second E2F-TDP domain, was not covered in our evolutionary analysis.

Evolution of RB family and DP family proteins in metazoa There are $36 \mathrm{RB}$ family proteins identified in 16 metazoan genomes (Figure 1). Both ML method and BI method were employed for phylogenetic analyses, RB-Mb (gi:167523296), the RB family protein from $M$. brevicollis, was used as an outgroup. Both methods produced nearly identical topologies (Figure 4). All the RB family proteins in Figure 4 were also summarized in Table 2.

RB family proteins were divided into RB1 subgroup and RBL subgroup with high statistical supports in metazoa (Figure 4). The placozoan, $T$ adhaerens, owns bone fide member RB1-Ta (gi:196012646) in RB1 subgroup, and RBL-Ta ((gi:196011866) in RBL subgroup. No-bilaterian animal sea anemone (N. vectensis) only possesses one gene of RB family, and was clustered into RBL subgroup in ML and BI phylogenetic analysis, Bilaterian, S. purpuratus and B. floridae, also possess their representative members in RB subgroup and RBL subgroup respectively. This indicated that the first gene duplication for ancient RB gene happened before the separation of placozoans and bilaterians. As the placozoans are thought to be diverged before the separation of cnidarians and bilaterians, the absence of RB1 subgroup orthologue in sea anemone ( $N$. vectensis) is possibly due to independent gene loss.

There are two RB family proteins, RBF1-Dm and RBF2-Dm, in D. melanogaster, and one RB family protein, Lin35, in C. elegans. None of them can be classed into any subgroup due to their high sequence divergence. As RBF1 and RBF2 are tightly clustered in our tree, we tend to think that RBF1 and RBF2 were formed by lineage specific gene duplication. Interestingly, we found that fly RBF1 gene share an interspecies conserved exon with Human RBL1 and RBL2 genes (Figure 3 , additional file 3 ), this data added the possibility that RBF1 belong to RBL subgroup. As the evidence is still limited, we do not class RBF1 into any subgroup in this study.

According to Figure 4, the second gene duplication occurred in RB family, but this only happened in RBL subgroup, not in RB1 subgroup. This gene duplication in RBL subgroup happened before tetrapoda and teleostei divergence, as $H$. sapiens and $D$. rerio possess representative RBL1 and RBL2 genes respectively. Human RBL1 and RBL2 gene emerged by gene duplications of the common RBL subgroup ancestor, and representative RBL subgroup members from invertebrates $T$. adhaerens, $N$. vectensis, S. purpuratus, $B$. floridae, and $C$. intestinalis, which we named them as RBL-Ta, RBL-Nv, RBL-Sp, RBL-Ci, and RBL-Bf, should be recognized as the co-orthologues of Human RBL1 and RBL2 genes (Table 2). Gene losses of RB family might also happen in some vertebrates. For example, RBL2 orthologue were not found in T. nigroviridis, and RB1 orthologue was not found in X. tropicalis. 


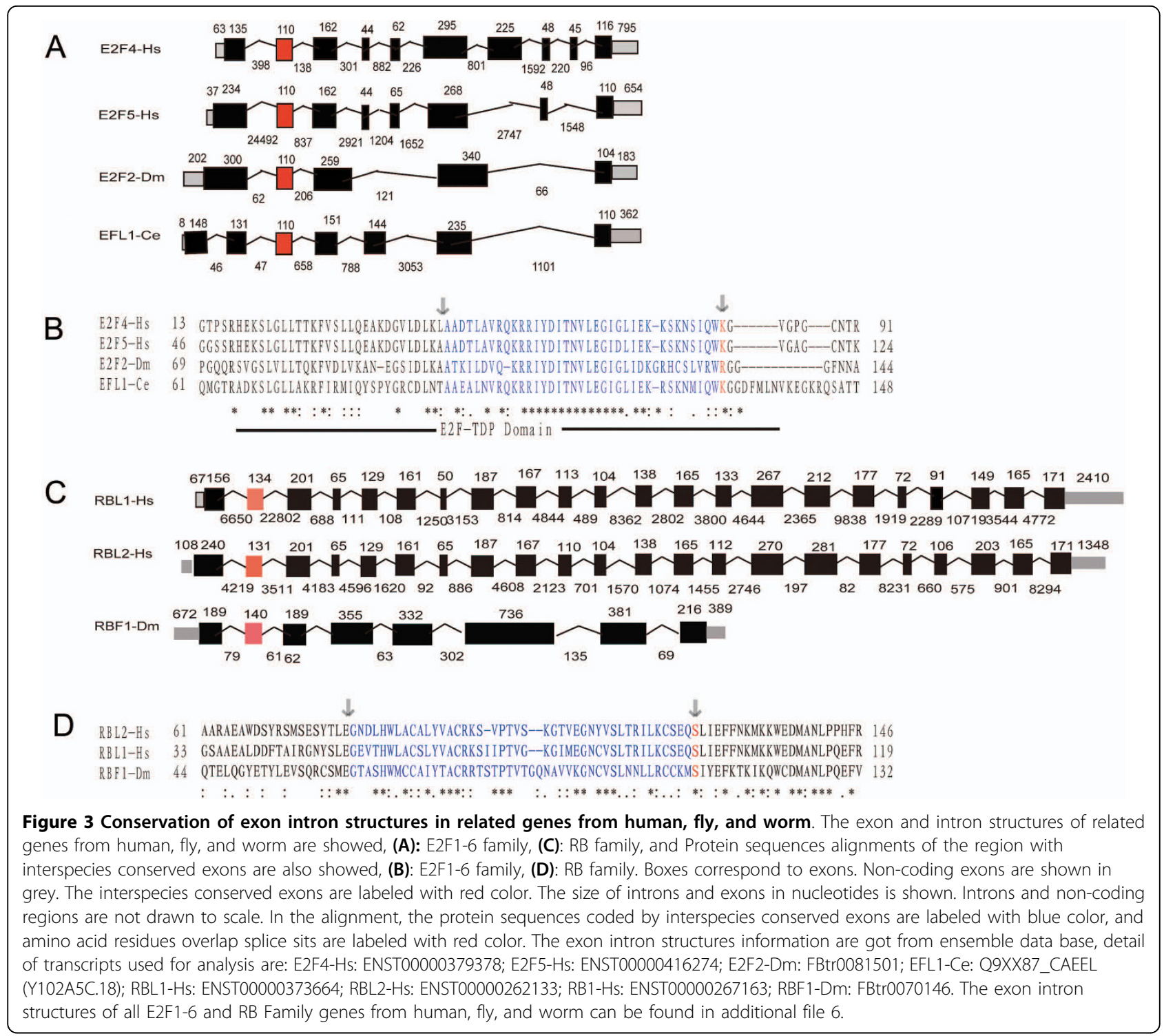

For DP family (Figure 4, Table 2), all invertebrates only have one orthologue of DP family. Similar to E2F7/ 8 family, gene duplication for DP family happened before tetrapoda and teleostei divergence. Incidentally, it was found that species-specific gene duplications for DP family happened in $H$. sapiens, M. musculus, and $D$. rerio. For example: Human DP3-Hs (gi:189409125) should be thought as the product of species-specific gene duplication of human DP1-Hs (gi:6005900).

\section{Repressive E2Fs (E2F4, E2F5) evolved slower than active E2Fs (E2F1, E2F2, E2F3)}

In $M L$ tree, the branch lengths for E2F1/2/3 subgroup proteins are longer than for E2F4/5 subgroup proteins in E2F1-6 protein family. This indicated E2F1/2/3 subgroup may evolve more rapidly than E2F4/5 subgroup.
So we did a detailed investigation of evolutionary rates of two subgroup proteins.

At first, the eukaryotic ancestral E2F sequence was predicted using the full alignment of E2F sequences of eukaryotic organisms and its phylogenetic tree by the software GASP. The ancestral E2F sequence could be found in additional File 4.

It was found that human E2F4 and E2F5 possess smaller distance to the predicted ancestral E2F sequence or the E2F-Mb (gi|167523471) compared with human E2F1, E2F2 and E2F3 (Figure 5A). However this kind of differences is not significant, as the small substitution rate difference could be partly masked by mutational saturation in comparisons between distantly related species. So we further investigated the substitute rate in E2F1/2/3 subgroup and E2F4/5 subgroup in more closely related organisms. 


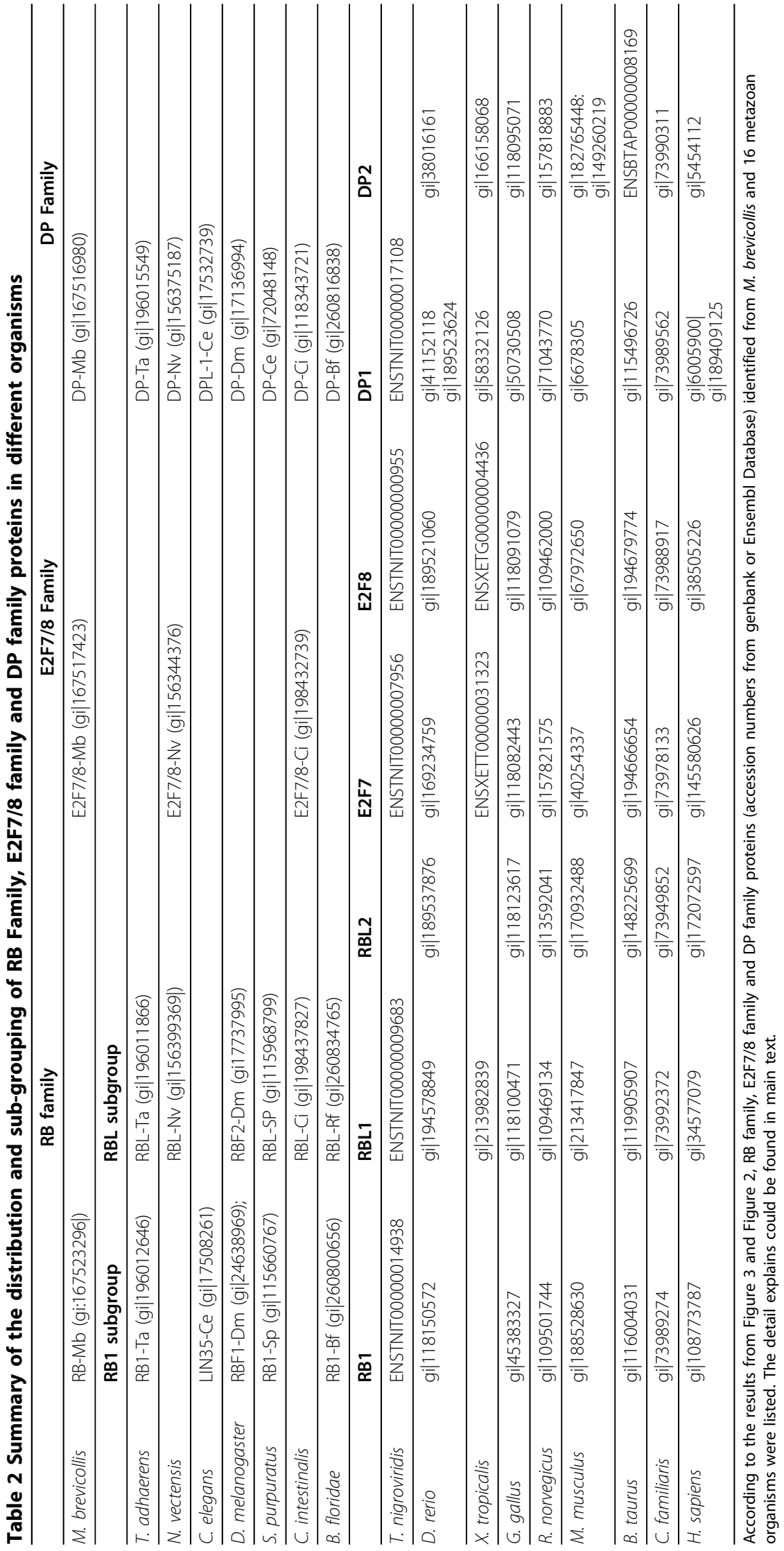




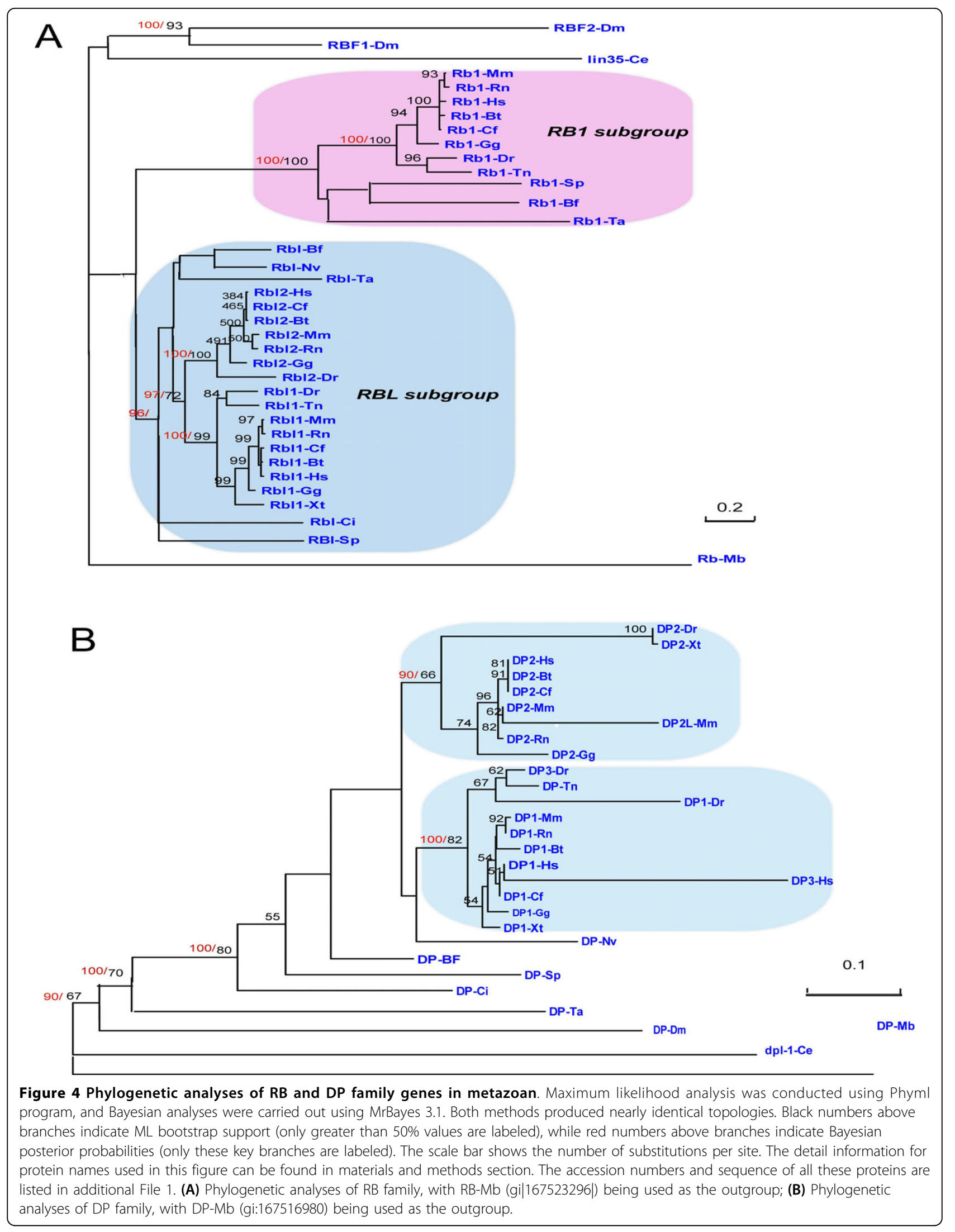




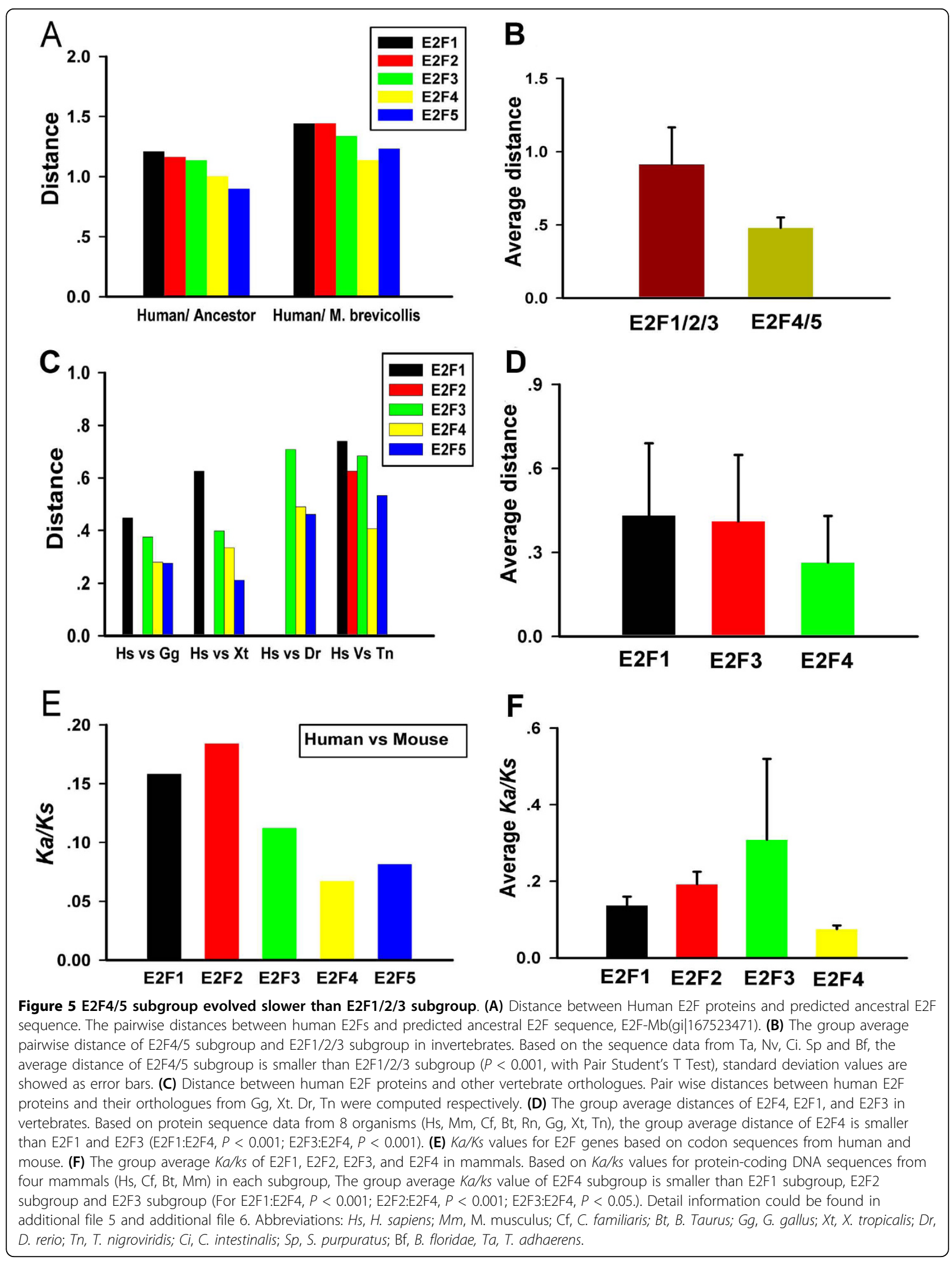


For invertebrates, based on the sequences data from 5 organisms ( $\mathrm{Ta}, \mathrm{Nv}, \mathrm{Sp}, \mathrm{Ci}$, and $\mathrm{Bf})$, we found that the group average pairwise distance among the orthologues in E2F4/5 subgroup were smaller than that in E2F1/2/3 subgroup $(P<0.001$, with pair Student's T Test) (Figure $5 B)$. In vertebrates, the distances between human E2F4, E2F5 and their orthologues from other vertebrates ( $G$. gallus; $X$ tropicalis; $D$. rerio; $T$. nigroviridis) were smaller than the distances between human E2F1, E2F2, E2F3 and their orthologues respectively (Figure. $5 \mathrm{C}$ ). As the group average pairwise distance comparisons between different genes general require these genes are derived from a same collection of organisms, Gene losses for E2F2 and E2F5 in several vertebrate organisms (Table 1) make E2F2 and E2F5 was not suitable to compute their group average pairwise distances and then make a statistical meaningful comparing in our study. For E2F1, E2F3, and E2F4 gene in vertebrate, the sequences data from 8 vertebrate organisms ( $\mathrm{Hs}, \mathrm{Mm}, \mathrm{Cf}, \mathrm{Bt}, \mathrm{Rn}, \mathrm{Gg}$, $\mathrm{Xt}, \mathrm{Tn}$ ) was used for analysis, we found that the average group pairwise distance of E2F4 gene is smaller than E2F1 and E2F3 $(P<0.001$, with Pair Student's T Test) (Figure 5D). In general, we thought the slower evolvement of E2F4/5 subgroup compared with E2F1/2/3 subgroup was conserved from invertebrates to vertebrates,

Finally, the selection pressures on human E2F proteins were examined by estimating the average ratio of nonsynonymous to synonymous substitutions $(\mathrm{Ka} / \mathrm{Ks})$ at each codon position. The codoning sequences alignments for E2F family proteins, and detail the $\mathrm{Ka} / \mathrm{ks}$ data could be found in additional file 5 and additional file 6 . We found that E2F1, E2F2 and E2F3 have severely reduced selection in comparison with E2F4 and E2F5 in cordon sequence comparison between human and mouse (Figure $5 \mathrm{E}$ ). In four mammals (Bt, $\mathrm{Hs}, \mathrm{Mm}$, and $\mathrm{Cf}$ ), we also found that the group average $\mathrm{Ka} / \mathrm{Ks}$ values for E2F4 was smaller than the group average $K a / K s$ values E2F1, E2F2, or E2F3 with statistical support $(P<0.05$, with pair Student's T Test) (Figure 5F). As E2F5 was absent in Bt and Rn, E2F5 was not covered in group average $K a / K s$ value study.

\section{RBL subgroup proteins evolved slower than RB1 subgroup proteins in vertebrates}

For RB family proteins, eukaryote ancestral RB sequence was predicted using the full alignments of all 39 RB like protein sequences of eukaryotic organisms and its phylogenetic tree by the software GASP. The ancestral E2F sequence could be found in additional File 3. However, differences in the distance between three human $R B$ family proteins and the ancestral RB sequence or RB$\mathrm{Mb}$ (gi:167523296) were small (Figure 6A).

In vertebrates, pairwise distances between human RB1, RBL1, RBL2 and their orthologues in G. gallus,
$X$. tropicalis, D. rario, $T$. nigroviridis were calculated. It was found that RB1 homologue evolved more rapidly compared with RBL1 homologue and RBL2 homologue (Figure 6B). Based on the sequence data from 6 organisms (Hs, Mm, Cf, Bt, Rn, Gg, and Dr), We find that the group average pairwise distance of RB1 gene was bigger than the group average pairwise distances of RBL1 gene or RBL2 gene $(P<0.001$, Pair T Test) (Figure $6 C)$.

To check the selection pressure, we first investigated $K a / K s$ values by sequences comparisons between human and other mammals (Figure 6D). The codoning sequences alignments for RB family proteins, and detail the $\mathrm{Ka} / \mathrm{ks}$ data could be found in additional file 5 and additional file 6. In 5 mammals (Bt, $\mathrm{Hs}, \mathrm{Mm}, \mathrm{Rn}$, and $\mathrm{Cf}$ ), the average $\mathrm{Ka} / \mathrm{ks}$ for RB1, RBL1, and RBl2 genes were also investigated (Figure 6E). In general, RB1 gene has higher $K a / K s$ ratios in comparison with RBL1 and RBL2 genes in mammals $(P<0.01$, pair T Test). Thus RB1 gene owns decreased selection pressure compared with RBL1 and RBL2.

\section{Discussion}

\section{Origination of the RB-E2F pathway and its possible} contribution for multicellular organisms emerging In our analysis, it was found that E2F1-6 family, RB family and DP family proteins are present in protist $D$. discoideum, green agar O. tauri, choanoflagellate M. brevicollis. This suggested that E2F and RB proteins were present in early ancestor of eukaryotes, and RB-E2F pathway originated in ancestor of eukaryotes before animal, plant and protist separation. One interesting finding in our study was that all multicellular organisms we checked owned orthologues from E2F1-6 family, RB family, and DP family, which indicated that the RB-E2F pathway is strongly conserved in multicellular organisms.

The emergence of multicellular organisms from single-celled ancestors marks one of the most pivotal events in life's history, which occurred several times, independently in different branches of the eukaryotic tree [30,31]. It was usually thought genes involved in cell-cell communication, cell adhesion and cell differentiation probably arose before, or concomitant with, the origins of multicellularity [30,31]. Given that RB-E2F pathway was found in all multicellular organisms we checked, we speculated this RB-E2F pathway might contribute to multicellular emergence. At least the core function of RB-E2F pathway (discussed in later) is consistent with this speculation. It was known that some cells may continue to proliferate and some cells may differentiate and give out its previous proliferate ability during the transition from unicellular organism to multicellular organism. The cycle controlling and/or differentiation function of RB-E2F clearly could contribute to 

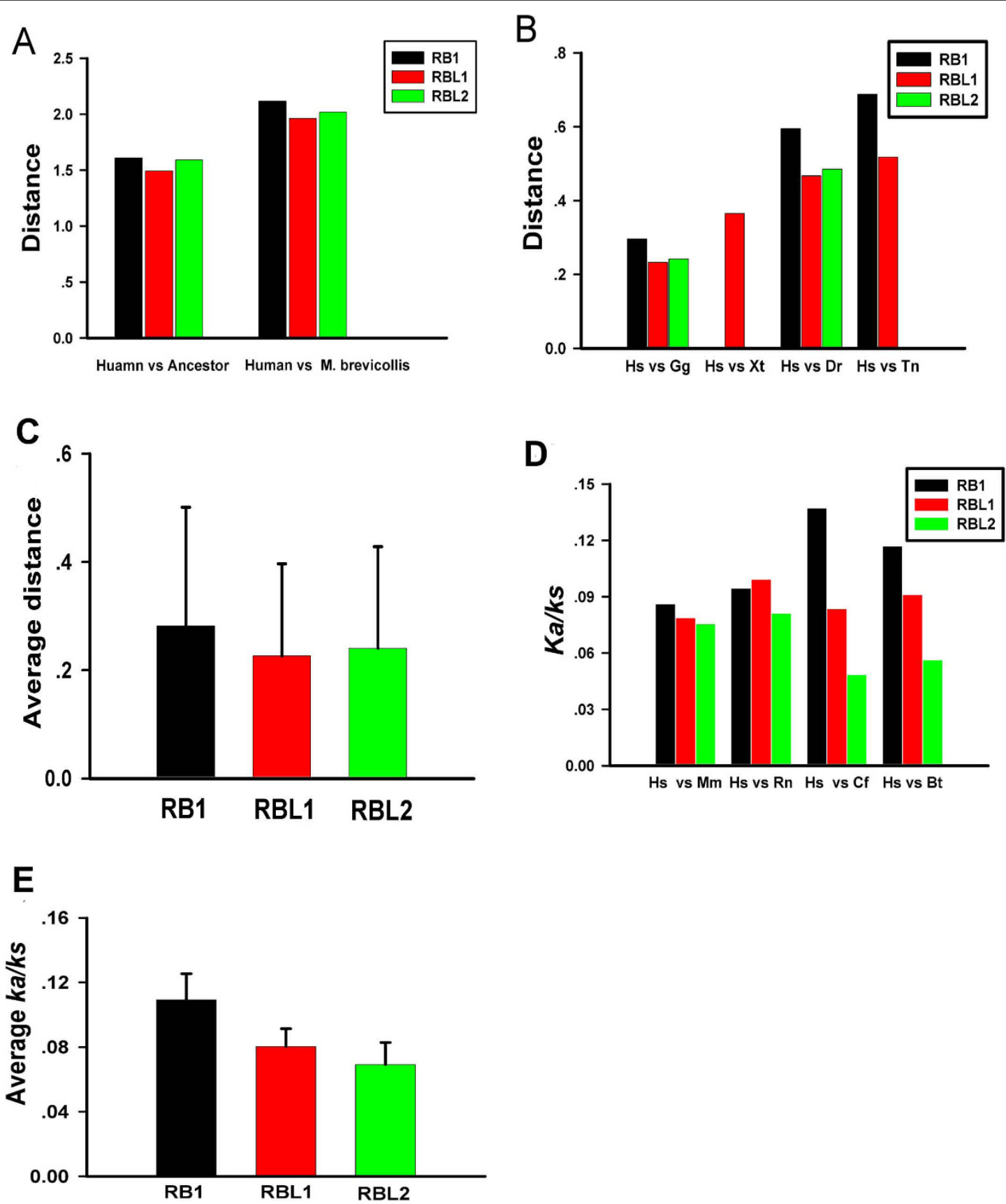

Figure 6 RBL subgroup evolved slightly slower than RB1 subgroup in vertebrates. (A) Distance between Human RB family proteins and predicted ancestral RB sequence. The pair wise distances between human RB1, RBL1, RBL2 and predicted ancestral RB sequence, RB-Mb (gi:167523296) sequence were computed. (B) Distance between human RB family proteins and their vertebrate orthologues. Pair wise distances were calculated between human RB1, RBL1, RBL2 and their orthologues in $\mathrm{Gg}, \mathrm{Xt}, \mathrm{Dr}, \mathrm{Tn}$, respectively. (C) The group average pairwise distances RB family in vertebrates. Based on protein sequences from 7 organisms ( $\mathrm{Hs}, \mathrm{Mm}, \mathrm{Cf}, \mathrm{Bt}, \mathrm{Rn}, \mathrm{Gg}$, and Dr), the group average distance of RBL1 subgroup and RBL2 subgroup is smaller than RB1 subgroup (For RB1: RBL1, $P<0.001$; RB1:RBL2, $P<0.001$; with pair Student's T test), standard deviation values are showed as error bars. (D) Ka/Ks values for RB family genes deduced from sequences comparisons between human and other mammals. The ratio of nonsynonymous (Ka) to synonymous substitutions (Ks) of RB1, RBI1 RBL2 genes were computed, based on codoning sequences pairwise comparisons (Hs vs Mm, Hs vs Rn, hs vs Cf, hs vs Bt) respectively. (E) Average Ka/ks values for RB family gene in mammals. Based on the Ka/ks values for all protein-coding DNA sequences from 5 mammals ( $\mathrm{Hs}, \mathrm{Cf}, \mathrm{Bt}, \mathrm{Mm}$, and $\mathrm{Rn}$ ) in each subgroup, The group average $\mathrm{Ka} / \mathrm{ks}$ value of RBL1 subgroup and RBL2 subgroup is smaller than RB1 subgroup with statistical support (For RBL1:RB1, $P<0.01$; RBL2:RB1, $P<0.01$ ), Detail information could be found in additional file 5 and additional file 6. Abbreviations used: Hs, H. sapiens; Mm, M. musculus; Cf, C. familiaris; Bt, B. Taurus; Gg, G. gallus; Xt, X tropicalis; Dr, D. rerio; Tn, T. nigroviridis; Ci, C. intestinalis; Sp, S. purpuratus; Bf, B. floridae. 
this progress. In fact, it was found that the expression of retinoblastoma orthologue was increased about 200 times when $D$. discoideum transited from unicellular to multicellular form [32]. By the way, beside E2F and RB proteins, some cycle regulatory components such as the KIP/WAF-type CDK inhibitor, Cyclin D, BRCA1 and BRCA2 are only found in plants and animals to the exclusion of the yeast [33].

\section{The ancient function of RB-E2F pathway: insights from its evolutionary history}

In this study, we mapped the evolutionary history of E2F and RB proteins. We summarized the evolutionary history of E2F1-6, RB, DP, and E2F7/8 family by listing their gene duplication events (Figure 7A). Ancestral E2F, RB genes duplicated before placozoans and bilaterians diverged, thus E2F family was divided into E2F4/5 subgroup and E2F1/2/3 subgroup, RB family was divided into RB1 subgroup and RBL subgroup (Figure 7A, Table 2). In vertebrates, the genes number in E2F1-6, RB, DP and E2F7/8 families increased by genes duplication events, these duplications occurred no later than tetrapoda and teleostei divergence (Figure 7A, Table 1, and Table 2). In general, we thought that the complexity increase of RB-E2F pathway is compatible with the increasing functional complexity in evolutionary history.

As mentioned in the results section, the fly dE2F1 was classed into E2F1/2/3 subgroup, and fly dE2F2 and worm EFL-1 was classed into E2F4/5 subgroup. This classification was consistent with results of previous functional studies [2]. As similar to repressive E2Fs (E2F4/5 subgroup), fly dE2F2 can form stable repressor complexes in conjunction with RBF1 and RBF2 [34], worm EFL-1 was also reported to form a stable repressor complex which represses the expression of many genes together with Lin35 [35]. And similar to active EFs, dE2F1 reverses the effects of dE2F2 repressor complexes [36]. As to fly RBF1, RBF2, and worm LIN35, as all of them have similar evolutionary distance to RB1 subgroup and RBL subgroup proteins, we do not classify them into any subgroup (Figure.7A).

One intriguing finding in our study was that E2F4/5 subgroup protein share more sequence similarity with the predicted E2F ancestral sequence than E2F1/2/3 subgroup proteins; E2F4/5 subgroup proteins evolved slower and own increased negative selection pressure than E2F1/2/3 subgroup. For RB family proteins, RBL subgroup proteins possess increased negative selection pressure compared with RB1 subgroup in mammals.

Protein evolutionary rates usually linked with protein function $[37,38]$. The rate of evolution of a protein-coding gene depends primarily on the structural-functional constraints that are intrinsic to the encoded protein $[37,38]$. As to duplication genes, in neofunctionalization model, it was thought that original copy gene maintain its ancestral function and keep the previous evolutionary rate, the new copy if not pseudogenized or lost, may acquire a new gene function. During this process of acquiring a new gene function, the new copy gene evolved faster than the original copy gene [39,40]. Thus, based on significantly different evolutionary rates within E2F family, we speculated that E2F4/5 subgroup proteins might mainly maintain the ancestral E2F function. And for RB family, RBL1 and RBL2 gene was under more strictly purification selection in mammals, we also speculated the ancestral RB family function might also mainly be represented by RBL subgroup proteins.

For RB-E2F pathway, it is general known that E2F1, E2F2 and E2F3 are active E2Fs and E2F4 and E2F5 are repressive E2Fs. RB family proteins are thought to function in cell cycle and cell differentiation. Though RB family might share similar functions, different RB family members might also function differently [2], only RB1 protein, but not RBL1 and RBL2, could interact to active E2Fs (E2F-1, E2F-2 and E2F-3); RBL1 and RBL2 can only interact to repressive E2Fs (E2F4 and E2F5) which is much more abundant and functional in G0-arrested cells than other E2F factors [2,41]. Interestingly, In $C$. elegans, there is only one RB family protein Lin-35, Lin 35 is somewhat more closely related to human RBL2 and RBL1 (Lin-35 shares 20,19, and 15\% overall aminoacid identity with RBL2, RBL1, and RB1, respectively) [42], previous studies did not reveal a cell-cycle role for Lin35, Lin35 is not rate limiting for S-phase entry [43]. In dictyostelium, the RB family orthologue also reported not function in cell cycle [32]. In Arabidopsis, local reduction of expression of the retinoblastoma-related (RBR) gene (homologue of human RB family) in roots increases the amount of stem cells without affecting cell cycle [44]. We speculated the ancient function of RB family was mainly related to cell differentiation, but not tightly related to cell cycle.

Functional studies in RB-E2F pathway also indicated that the function conducted by E2F4/5 subgroup and RBL subgroup proteins is extremely conserved. A key finding about RB-E2F pathway came with the biochemical purification of native E2F-RBF complexes from $D$. melanogaster embryo extracts (dREAM [45] and MybMuvB (MMB) [46]), and the repressive dE2F2, RBF1 and RBF2 are members of these complexes. Similar complexes were subsequently purified from C. elegans (DRM [47]), which contain Lin35 and EFL-1. For mammals, similar complex was also found (DREAM $[48,49]$ and IINC [50]). These complexes contain the repressive E2F protein E2F4 or E2F5, and RBL subgroup protein RBIL1 or RBL2. Human DREAM was found to bind to more than 800 human promoters in G0 and was required for repression of E2F target genes [49]. As to 


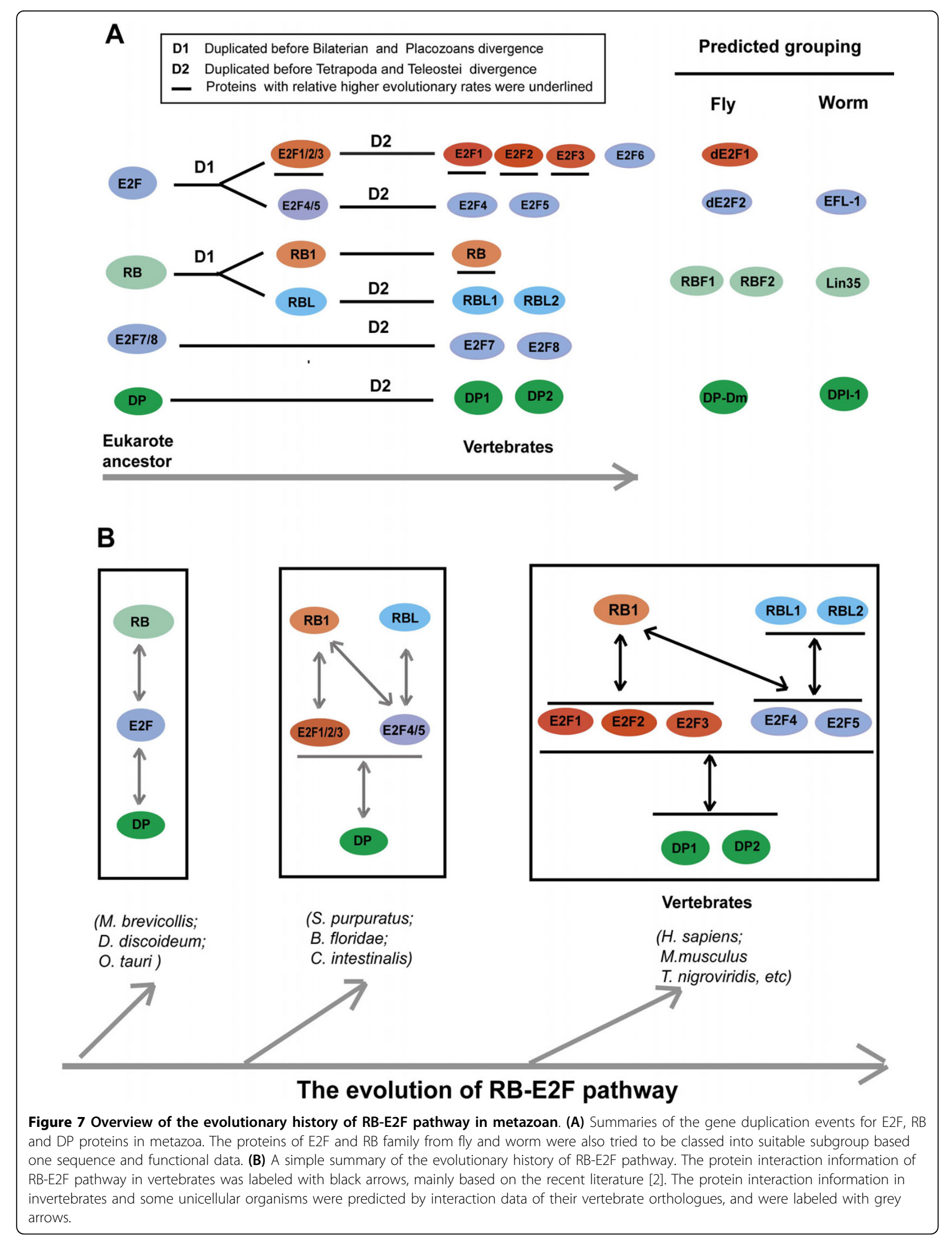


the E2F1/2/3 subgroup and RB1 subgroup members, no similar conserved protein complex was found till now.

Taken all together, it was temptation to think that the function conducted by E2F4/5 subgroup and RBL subgroup might be more conserved than those function conducted by E2F1/2/3 subgroup and RB1 subgroup.

\section{Conclusions}

The most important cell fate decisions, such as whether to divide, differentiate or die, usually are very strictly regulated. The RB-E2F pathway plays critical roles on these fundamental cellular processes. We carried out comprehensive evolutionary analyses for RB and E2F family, our data demonstrated that E2F4 and E2F5 share more sequence similarity with the predicted E2F ancestral sequence than E2F1, E2F2 and E2F3; E2F4 and E2F5 also possess significantly lower evolutionary rates and higher negative selection pressures than E2F1, E2F2 and E2F3. For RB family, the RBL subgroup proteins evolved slower and also possess higher purification selection pressures compared with RB1 subgroup proteins. We speculated that that ancient function of RBE2F pathway might mainly link with repressive E2F and RBL subgroup proteins. And the active E2F and RB1 subgroup proteins might contribute more to functional diversification in RB-E2F pathway. Our results will enhance the current understanding of RB-E2F pathway and will also be useful to further functional studies in human and other model organisms.

\section{Reviewer's report 1}

Pierre Pontarotti (UMR 6632 Université de Aix Marseille/ CNRS. Equipe Evolution biologique et Modélisation France)

This article describes the phylogenetic analysis of three gene families involved in the same pathway. The robustness of the phylogenetic analysis is proper, however I disagree with the title of the article that do not reflect exactly the work carried out. Indeed the authors performed only a classification. However I think that this classification of protein families is helpful for the scientific community and thus I recommend the paper to be published.

Author's response: As the article also discussed much about the ancient function of RB-E2F pathway, we still kept the title "The ancient function of RB-E2F Pathway: insights from its evolutionary history"

\section{General remark}

The paper is difficult to understand and should be clarified, in particular the fact that some duplications are lineage-specific and other are older. The authors should use terms such as in paralog or co-orthologs to make the information clearer. I think that the paper should be reworked and the concept and result clarified. I wonder also if the authors should propose a new nomenclature, take as example this article: "Nme protein family evolutionary history, a vertebrate perspective by Desvignes et al 2009". This will clarify the issue.

Author's response: We agree that it is a little difficult to understand for some evolutionary relationship in E2F1-6 family and RB family, as there were ancient gene duplications and lineage-specific gene duplications in E2F1-6 family and RB family. We use the term co-orthologues to describe some evolutionary relationships in the revision, for example, E2F4/5 subgroup members from invertebrates $T$ adhaerens, E2F4/5-Ta, should be recognized as the co-orthologues of vertebrate E2F4 and E2F5 gene. We added two tables, similar to the article: "Nme protein family evolutionary history, a vertebrate perspective by Desvignes et al 2009" [51].

In several instances, the phylogeny based only on bases substitution could not be informative, therefore other characters should be taken into an account, for example exon intron organization (see for example page 10, third paragraph).

Author's response: $E 2 F$ and $R B$ family protein sequences from fly and worm usually diverged highly and could not be clustered into subgroup in phylogenetic analysis based on protein sequences. As the reviewer's suggestion, The Exon intron structure of E2F1-6 family, $R B$ family genes from fly, worm, and human were studied in this revision (figure 3 , additional file 3). We found that E2F2-Dm, E2FL1-Ce, E2F4-hs, and E2F5-hs gene share some Exon intron structure similarity, which support our speculation that E2F2-Dm and E2FL1-ce gene should be classified into E2F4/5 subgroup. However, For E2F1-Dm gene, no Exon intron structure similarity with human E2F1/2/3 subgroup or E2F4/5 subgroup genes were found. For RB family, we found that fly RBF1 gene share an interspecies conserved exon with Human RBL1 and RBL2 genes (Figure 3), these data added the possibility that RBF1 belong to RBL subgroup. As the evidence is still limited, we do not class RBF1 into any subgroup in this study.

My major problem concerned the figure 6 in which the author indicate that for example that B Floridae is the vertebrate ancestor. The entire lineage evolved, some show, of course, more apomorphism or plesiomorphism than other, However none of the present day specie is the ancestor of another present day specie.

Author's response: We corrected these problems in Figure 6 (Now is Figure 7).

The sentence page 11: "during the transition from invertebrate to vertebrate" is also misleading. The authors should write a second gene duplication occurred in the RB family in the vertebrate lineage after their separation with the amphioxus ancestor.

Author's response: We corrected these problems. We changed it to "The second gene duplication occurred in 
$R B$ family, but this only happened in RBL subgroup, not in RB1 subgroup. This gene duplication in RBL subgroup happened before tetrapoda and teleostei divergence, as $H$. sapiens and D. rerio possess representative RBL1 and RBL2 genes respectively"

\section{Other comments}

Lot of misspellings are present in this report. This start with the first line of the paper where the word background is misspelled. In general vocabulary and grammar should be checked by a native English speaker. Please ask to a native Anglophone to review the article All the phylogenetic trees presented in the figures show problems with the species branching For example; figure 1 E2F1 of Xenopus and Tetraodon form a monophylogenetic group; instead Xenopus should branch with Gallus and mammalian. Moreover, the authors have to check all the phylogenetic incongruence and comment them.

Author's response: We corrected several misspellings which have been mentioned by three reviewers and also asked one native English speaker to review this article. We corrected the mistake about "Xenopus and Tetraodon form a monophylogenetic group".

The phylogenetic incongruences sometimes are due to protein sequence highly diverged in some organisms (such as in S. purpuratus; C. intestinalis; C. elegans; and D. melanogaster). We added some comments for these phylogenetic incongruences in the revision. For example, According to Dr. Arcady Mushegian's suggestion, we added the protein sequences from the placozoan $T$. adhaerens in our analysis. T. adhaerens is a basal eumetazoan lineage that diverged before the separation of cnidarians and bilaterians. As $T$ adhaerens owns representative members for $R B 1$ subgroup and $R B L$ subgroup. And N. vectensis possess representative member in RBL1 subgroup, but no member in RB1 subgroup. Based on the data in T. adhaerens, we speculated that this absence of orthologue of RB1 subgroup in $N$. vectensis is due to independent gene loss.

\section{Reviewer's report 2}

\section{Dr. Arcady Mushegian (Stowers Institute, Kansas City,} United States)

p.3 "Proteins that are related to the retinoblastoma tumor suppressor RB and the E2F transcription factor... have been missing from yeasts and other fungi" - this is incorrect. Orthologs of retinoblastoma may be missing from the fungi, but "proteins related" to retinoblastoma are certainly there, i.e., any protein that contains a BRCT domain.

Author's response: We corrected this mistake, and we mentioned that some fungi possess orthologues of E2F1-6 family and DP family, but no orthologue of RB family in the introduction. p.5 E-value less than e-5 was used to collect the homologs. At a more common default value of e-3, have there been more matches, especially in the species that the authors say lacked any homologs (e.g., fungi)?

Author's response: As reviewer's suggestion, using human E2F4, E2F7, DP1, RB1 proteins, we performed Blast searchings, with E-value less than e-3 as cut-off, in NCBI data base. We got the same results in the organisms we checked. For Fungi, I will answer it below.

The authors do not mention Trichoplax adhaerens at all. This is a shortcoming: even if Placozoa are not the most primitive metazoan animal, it is still primitive clade, perhaps close to the split of cnidaria and bilateria. All hypotheses about what happened before or after the latter split may gain additional evidence if this species is included (genes are not very well annotated in Trichoplax, so some prediction by homology may be needed).

Author's response: Thanks for the good suggestion. We found Trichoplax adhaerens own two orthologues of E2F1-6 family, and two orthologues of RB family, and one orthologue of DP family. T. adhaerens are thought to be diverged before the separation of cnidarians and bilaterians [12]. E2F and RB family proteins from $T$. adhaerens are now covered in analysis in the revision.

I would be happier if multiple fungi with complex morphology were included in the analysis, not only yeasts with what we know to be abbreviated genomes.

Author's response: We did the Blast searching, with human E2F4, E2F7, RB1, and DP as queries, in fungi (taxid:4751) in NCBI database. In addition, domain associations of all possible proteins were checked in SMART and pfam database. In total, we found six proteins which belong to E2F1-6 family or DP family in fungi. Below is the detail: Nosema ceranae BRL01 owns one E2F1-6 family protein (gi:239605701), one DP family protein (gi:239605391); Enterocytozoon bieneusi H348 owns one E2F1-6 family protein gi|169806750), one DP family protein (gi|169806250); Encephalitozoon cuniculi GB-M1 owns one E2F1-6 family protein gi|19074054), and one DP family protein (gi|19074276), However, No $R B$ family protein was found in fungi. N. ceranae, E. bieneusi, and E. cuniculi belong to the phylum Microsporidia, The microsporidia constitute a phylum of spore-forming unicellular parasites. They were once thought to be protists but are now known to be fungi [10].

As our main interest is on RB-E2F protein evolution in metazoa, and no RB family protein was found in fungi, we did not cover these fungi E2F and DP protein in detail analysis this time. However, we mentioned the E2F1-6 family, and DP family proteins from E. cuniculi in the introduction in this revision.

p.10, par.3 replace "homologue" with "orthologoue" twice? All E2F family members are homologs by 
definition. Similarly elsewhere in the manuscript, for example the fourth paragraph on the same page.

Author's response: we correct them and similar problems in elsewhere in the manuscript

p.16-17: "based on the significantly evolutionary rate differences [should be: significantly different evolutionary rates] within E2F family and RB family, we speculated that E2F4/5 subgroup proteins might mainly maintain the ancestral E2F function. And for RB family, the ancestral RB family 17 function might also mainly represent by RBL subgroup proteins." - What those ancestral functions might be, as opposed to derived functions of the other family members? This is answered very briefly in penultimate paragraph on p. 17, but needs to be elaborated.

Author's response: we change to "significantly different evolutionary rates" as reviewer suggested, We add one paragraph which was tried to describe about function difference between RB1 subgroup and RBL subgroup, and speculated that ancient function of $R B$ family might be linked with cell differentiation but not with cell cycle controlling.

\section{Reviewer's report 3}

Dr. Zhenguo Lin, Department of Ecology and Evolution, The University of Chicago (nominated by Dr. Neil Smalheiser)

The RB-E2F pathway is involved in several fundamental processes of cellular activities, including regulating the initiation of DNA replication. Disruption of the RB-E2F pathway has been shown to be associated with all human tumors. Therefore, it is of great interest to study the origin and evolution of this pathway. The authors reconstructed the evolutionary histories for the three gene families (E2F, RB and DP) in this pathway. They also found that different members in each of the RB and E2F family might have experienced different selection constraints. This study provides some new understandings about this important pathway.

\section{Major comments}

One of the major conclusions of this study is that "E2F4/5 genes have "significantly lower evolutionary rates" than E2F1/2/3, and RBL1/2 "also evolved slightly slower compared with RB1 in vertebrates". These conclusions are based on comparing values of protein divergence data or $K a / K s$. I do not know what criteria were used to determine if the evolutionary rate is "significantly lower" or "slightly slower". For example, the authors also used "this kind of differences is not big," "no big difference was found in the distance" in Page 13. Without any appropriate statistical inference, such claims seem to be invalid. In addition, the authors only compared human with other species to obtain protein distances and $K a / K s$. I would suggest authors to calculate all pairwise distance values for all protein sequences in each subgroup, and perform a statistical evaluation to determine if two subgroups evolve under significant different rates.

Author's response: As the reviewer's suggestion, we did group average pairwise distance value comparisons, and group average Ka/ks value comparisons, and perform statistical evaluations.

For group average pairwise distance values comparisons, all pairwise distance values for all protein sequences in each subgroup was first computed. Then average value of group pairwise distances was calculated. For group average Ka/ks values comparing, all Ka/ks values for all protein-coding DNA sequences in each subgroup was first computed. Pair Student's T Test was used for statistical analysis. Considering the effect of gene losses, we thought that the group average pairwise distances comparing between different genes general require these genes are derived from a same collection of organisms. For E2F1-6 family, we did group average pairwise distances comp comparison invertebrates, (Figure 5B), and in vertebrate (Figure 5D), For RB family, we did group average pairwise distances comp comparison in vertebrate (Figure 6C), For group average Ka/ks values comparison, we did them in E2F1-6 family (Figure 5F), and in RB family (Figure 6E). In the new additional file 6, all the data about group average distances and group average ka/ks values could be found.

In general, the E2F4/5 subgroup evolved significantly slower than E2F1/2/3 subgroup $(P<0.001$, with pair $T$ Test), For RB family, RBL subgroup proteins evolved slower than $R B 1$ subgroup proteins in vertebrates $(P<$ 0.01, with Pair T Test)

The basal groups on the tree of E2F1-6 subfamily in Fig 2A and Fig S3A are poorly resolved, therefore the evolutionary relationships between the Monosiga brevicollis E2F genes with other E2F member remains unclear. Under this condition, $M b E 2 F$ gene could be a member of either E2F1/2/3 group or E2F4/5 groups. So, I am not sure if it is appropriate to use MbE2F as the outgroup to infer that E2F1/2/3 group or E2F4/5 groups.

Author's response: One reason for our using Monosiga brevicollis E2F as the outgroup of Metazan E2F1-6 family was that $M$. brevicollis is the close relative of metazoa, And we had tried many times, but we still failed to classified $M$. brevicollis E2F genes into either E2F1/2/3 group or E2F4/5 groups. When the E2F protiens from $D$. discoideum or plant were used as outgroups, the topology of E2F1/2/3 group or E2F4/5 groups in metazoa do not change comparing with E2F from $M$. brevicollis was used as the outgroup.

E2F1/2/3-Nv (gi:156371340) sequence is much shorter than other E2F proteins, it contains only the $\mathrm{N}$-terminal 
domains and it is about $1 / 2$ length of its orthologous genes. The prediction of its CDS is probably incorrect. I would suggest the authors to do TBLASTN against its genomic sequence to obtain its complete sequence. Otherwise, it is not a good idea to include partial sequence in the alignment for tree building. In addition, E2F3-Rn has the same problem. The authors need to carefully inspect the alignments to detect obscure sequences. Better trees are needed for better supports of their conclusions.

Author's response: We tried to do TBLASTN against its genomic sequence using the E2F1/2/3-Ta (gi:196010483) which is most similar to E2F1/2/3-Nv (gi:156371340) in our analysis, But still good result. As E2F1/2/3(gi:156371340) sequence contained the E2FTDP domain, and still be good cluster into E2F1/2/3 subgroup, so We kept it in analysis, For E2F3-Rn, We rechecked it sequences in genbank and in ensembl data base, In ensembl database, Rat E2F3 gene, LOC691420 (ENSRNOT00000050261), also only coded 245 AA. When we did two sequence blast for mRNA sequences of Human E2F3 (GI:16848011) and Rat E2F3 (GI:212549626), We found that a reading frame shift for Rat E2F3 which induced rat E2F3 protein sequence had been unexpected stopped in comparison with human E2F3 mRNA.. In detail: the sequence at the position 1231-1254 of human E2F3 mRNA (GI:168480112) is "CAA-GAT-ATT-CGA-AAA-ATT-AGT-GGC", the sequence at the position 1045-1068 of Rat E2F3 mRNA (GI:212549626) is "TCA-AGA-TAT-TCG-AAA-AATTAG-TGG".

\section{Minor comments}

Page 5. "in additional" should be "in addition", or "additionally".

Author's response: we corrected it.

Page 8. First line" We found that all three unicellular organisms, D. discoideum AX4, O. tauri, and M.brevicollis contain". In fact, S. pombe and S. cerevisiae are also unicellular organisms.

Author's response: The fact is that some unicellular organisms owns E2F, RB family protein, and some unicellular organisms (S. pombe and S. cerevisiae) not. We removed the word "all".

Page 8. Last line of first paragraph. "E2F7/8 family was not as indispensable as E2F1-6, RB, and DP family" if it is not your conclusion, reference is needed.

Author's response: it is our conclusion.

Page 9, Second paragraph "did not function in classical RB-E2F pathway" any reference?

Author's response: we added the reference.

Page 10, third paragraph "And this classification was also consistent with results of previous functional studies." any reference?

Author's response: we added the reference.
Page 10, third paragraph "its sequence highly diverged" should be "its sequence IS highly diverged".

Author's response: we corrected it.

Page 11. "RBF1 and RBF2 are formed by lineage specific gene duplication, and this gene duplication may not happen very early" Only one arthropod species (fruit fly) was used in this study, I agree that RBF1 and RBF2 are formed by lineage specific gene duplication, but I am not sure about "not happen very early". The two branches seem to be long; the duplication could happen as early as before divergence of arthropods. Therefore, without including more arthropod species, this conclusion seems to be under question.

Author's response: we corrected it, and removed " and this gene duplication may not happen very early"

Page 14 "partly" should be "partially"

Author's response: we corrected it.

Figure legend, Fig 4. "Codon sequence", I think it is better to use "Coding sequences"

Author's response: we corrected it.

\section{Additional material}

Additional file 1: E2F1-6, E2F7-8, RB, and DP family proteins sequences. The protein name and Genbank accession number for all of E2F1-6, E2F7-8, RB, and DP family proteins we identified in this study. The sequence of all E2F1-6, E2F7-8, RB, and DP family proteins were also listed.

Additional file 2: Phylogenetic analyses of E2F1-6, E2F7/8, RB, and DP family in eukaryote. Maximum likelihood $(\mathrm{ML})$ trees were constructed by using PHYML V.2.4 for E2F1-6, E2F7/8, RB, and DP family in eukaryota, with 200 bootstrap resamplings and JTT setting,

Additional file 3: Exon intron structures. The exon and intron structures of E2F and RB family genes from human, fly, and worm. Additional file 4: Predicted ancestral sequences of E2F and RB. Detail ancestral sequences of E2F and RB predicted by Gapped Ancestral Sequence Prediction program (GASP) program

Additional file 5: The codoning sequences alignments for E2F and RB family proteins. The codoning sequences alignments for E2F and RB family proteins used in computing $\mathrm{Ka} / \mathrm{Ks}$ in Figure 5 and Figure 6.

Additional file 6: The protein pairwise distance and the $K a / k s$ data The detail data of the protein pairwise distance and the $\mathrm{Ka} / \mathrm{ks}$ Values used for group comparisons in this study.

\section{Abbreviations}

HS: Homo sapiens; CF: Canis familiaris; BT: BOS TAURUS; MM: Mus musculus; RN: Rattus norvegicus; GG: Gallus gallus; XT: Xenopus tropicalis; DR: Danio rerio; TN: Tetraodon nigroviridis; SP: Strongylocentrotus purpuratus; Bf: Branchiostoma floridae; Cl: Ciona intestinalis; CE: Caenorhabditis elegans; DM: Drosophila melanogaster; NV: Nematostella vectensis; Ta: Trichoplax adhaerens; Mb: Monosiga brevicollis: At: Arabidopsis thaliana; Os: Oryza sativa; Dd: Dictyostelium discoideum AX4; Ot: Ostreococcus tauri.

\section{Acknowledgements}

We are appreciated for the helpful comments from three reviewers (Dr. Pierre Pontarotti, Dr. Arcady Mushegian and Dr. Zhenguo Lin). And we also are grateful to nomination of Dr. Neil Smalheiser, This work was supported by National 863 project of China [2006AA020501]; National Key Sci-Tech Special Project of China [2008ZX10002-020]. 


\section{Authors' contributions}

LC and LY conceived this study; LC, BP, MZ, and KS collected the data; LC, $\mathrm{BP}$ and $\mathrm{LY}$ made the analysis; $\mathrm{LC}, \mathrm{BP}$ and $\mathrm{MY}$ wrote the manuscript.

\section{Competing interests}

The authors declare that they have no competing interests.

Received: 12 May 2010 Accepted: 20 September 2010

Published: 20 September 2010

\section{References}

1. Weinberg RA: The Retinoblastoma Protein and Cell-Cycle Control. Cell 1995, 81(3):323-330.

2. van den Heuvel S, Dyson NJ: Conserved functions of the pRB and E2F families. Nature Reviews Molecular Cell Biology 2008, 9(9):713-724.

3. laquinta PJ, Lees JA: Life and death decisions by the E2F transcription factors. Current Opinion in Cell Biology 2007, 19(6):649-657.

4. Polager S, Ginsberg D: E2F-at the crossroads of life and death. Trends in Cell Biology 2008, 18(11):528-535.

5. Trimarchi JM, Fairchild B, Verona R, Moberg K, Andon N, Lees JA: E2F-6, a member of the E2F family that can behave as a transcriptional repressor. Proc Natl Acad Sci USA 1998, 95(6):2850-2855.

6. Cam H, Dynlacht BD: Emerging roles for E2F: Beyond the G1/S transition and DNA replication. Cancer Cell 2003, 3(4):311-316.

7. Chen $\mathrm{HZ}$, Tsai SY, Leone G: Emerging roles of E2Fs in cancer: an exit from cell cycle control. Nature Reviews Cancer 2009, 9(11):785-797.

8. Iyer $L$, Anantharaman V, Wolf $M$, Aravind $L$ : Comparative genomics of transcription factors and chromatin proteins in parasitic protists and other eukaryotes. International Journal for Parasitology 2008, 38(1):1-31.

9. Claudio PR, Tonini T, Giordano A: The retinoblastoma family: twins or distant cousins? Genome Biol 2002, 3(9), reviews3012.

10. Takemura M: Evolutionary history of the retinoblastoma gene from archaea to eukarya. Biosystems 2005, 82(3):266-272.

11. Lee SC, Corradi N, Byrnes lii EJ, Torres-Martinez S, Dietrich FS, Keeling PJ, Heitman J: Microsporidia Evolved from Ancestral Sexual Fungi. Current Biology 2008, 18(21):1675-1679.

12. Srivastava M, Begovic E, Chapman J, Putnam NH, Hellsten U, Kawashima T, Kuo A, Mitros T, Salamov A, Carpenter ML, Signorovitch AY, Moreno MA, Kamm K, Grimwood J, Schmutz J, Shapiro H, Grigoriev IV, Buss LW, Schierwater B, Dellaporta SL, Rokhsar DS: The Trichoplax genome and the nature of placozoans. Nature 2008, 454(7207):955-960.

13. Putnam NH, Srivastava M, Hellsten U, Dirks B, Chapman J, Salamov A, Terry A, Shapiro H, Lindquist E, Kapitonov W, Jurka J, Genikhovich G, Grigoriev IV, Lucas SM, Steele RE, Finnerty JR, Technau U, Martindale MQ, Rokhsar DS: Sea anemone genome reveals ancestral eumetazoan gene repertoire and genomic organization. Science 2007, 317(5834):86-94.

14. King N, Westbrook MJ, Young SL, Kuo A, Abedin M, Chapman J, Fairclough S, Hellsten U, Isogai Y, Letunic I, Marr M, Pincus D, Putnam N, Rokas A, Wright KJ, Zuzow R, Dirks W, Good M, Goodstein D, Lemons D, Li W, Lyons JB, Morris A, Nichols S, Richter DJ, Salamov A, Sequencing JG, Bork P, Lim WA, Manning G, Miller WT, McGinnis W, Shapiro H, Tjian R, Grigoriev IV, Rokhsar D: The genome of the choanoflagellate Monosiga brevicollis and the origin of metazoans. Nature 2008, 451(7180):783-788.

15. Eichinger $L$, Pachebat JA, Glockner $G$, Rajandream MA, Sucgang $R$, Berriman M, Song J, Olsen R, Szafranski K, Xu Q, et al: The genome of the social amoeba Dictyostelium discoideum. Nature 2005, 435(7038):43-57.

16. Derelle E, Ferraz C, Rombauts $S$, Rouze P, Worden AZ, Robbens $S$, Partensky F, Degroeve S, Echeynie S, Cooke R, Saeys Y, Wuyts J, Jabbari K, Bowler C, Panaud O, Piégu B, Ball SG, Ral JP, Bouget FY, Piganeau G, De Baets B, Picard A, Delseny M, Demaille J, Van de Peer Y, Moreau H: Genome analysis of the smallest free-living eukaryote Ostreococcus tauri unveils many unique features. Proc Natl Acad Sci USA 2006, 103(31):11647-11652.

17. Altschul SF, Madden TL, Schaffer AA, Zhang JH, Zhang Z, Miller W, Lipman DJ: Gapped BLAST and PSI-BLAST: a new generation of protein database search programs. Nucleic Acids Res 1997, 25(17):3389-3402.

18. Letunic I, Copley RR, Pils B, Pinkert S, Schultz J, Bork P: SMART 5: domains in the context of genomes and networks. Nucleic Acids Res 2006, 34 D257-D260.
19. Finn RD, Tate J, Mistry J, Coggill PC, Sammut SJ, Hotz HR, Ceric G, Forslund K, Eddy SR, Sonnhammer EL, Bateman A: The Pfam protein families database. Nucleic Acids Res 2008, 36:D281-D288.

20. Edgar RC: MUSCLE: multiple sequence alignment with high accuracy and high throughput. Nucleic Acids Res 2004, 32(5):1792-1797.

21. Thompson JD, Gibson TJ, Plewniak F, Jeanmougin F, Higgins DG: The CLUSTAL_X windows interface: flexible strategies for multiple sequence alignment aided by quality analysis tools. Nucleic Acids Res 1997, 25(24):4876-4882.

22. Guindon S, Gascuel O: A simple, fast, and accurate algorithm to estimate large phylogenies by maximum likelihood. Syst Biol 2003, 52(5):696-704.

23. Schmidt HA, Strimmer K, Vingron M, von Haeseler A: TREE-PUZZLE: maximum likelihood phylogenetic analysis using quartets and parallel computing. Bioinformatics 2002, 18(3):502-504

24. Ronquist $F$, Huelsenbeck JP: MrBayes 3: Bayesian phylogenetic inference under mixed models. Bioinformatics 2003, 19(12):1572-1574.

25. Edwards RJ, Shields DC: GASP: Gapped ancestral sequence prediction for proteins. Bmc Bioinformatics 2004, 5:123.

26. Kumar S, Tamura K, Nei M: MEGA3: Integrated software for Molecular Evolutionary Genetics Analysis and sequence alignment. Briefings in Bioinformatics 2004, , 5: 150-163.

27. Suyama M, Torrents $D$, Bork P: PAL2NAL: robust conversion of protein sequence alignments into the corresponding codon alignments. Nucleic Acids Res 2006, 34:W609-W612.

28. Yang ZH: PAML 4: Phylogenetic analysis by maximum likelihood. Mol Biol Evol 2007, 24(8):1586-1591.

29. Nei M, Gojobori T: Simple Methods for Estimating the Numbers of Synonymous and Nonsynonymous Nucleotide Substitutions. Mol Biol Evol 1986, 3(5):418-426.

30. Rokas A: The Origins of Multicellularity and the Early History of the Genetic Toolkit For Animal Development. Annual Review of Genetics 2008, 42:235-251.

31. Ruiz-Trillo I, Burger G, Holland PWH, King N, Lang BF, Roger AJ, Gray MW: The origins of multicellularity: a multi-taxon genome initiative. Trends in Genetics 2007, 23(3):113-118.

32. MacWilliams $H$, Doquang $K$, Pedrola R, Dollman G, Grassi D, Peis T, Tsang A Ceccarelli A: A retinoblastoma ortholog controls stalk/spore preference in Dictyostelium. Development 2006, 133(7):1287-1297.

33. Aravind L, Subramanian G: Origin of multicellular eukaryotes - insights from proteome comparisons. Current Opinion in Genetics \& Development 1999, 9(6):688-694.

34. Dimova DK, Stevaux O, Frolov MV, Dyson NJ: Cell cycle-dependent and cell cycle-independent control of transcription by Drosophila E2F/RB pathway. Gene Dev 2003, 17(18):2308-2320.

35. Lu XW, Horvitz HR: lin-35 and lin-53, two genes that antagonize a Celegans Ras pathway, encode proteins similar to $\mathrm{Rb}$ and its binding protein RbAp48. Cell 1998, 95(7):981-991.

36. Frolov MV, Huen DS, Stevaux O, Dimova D, Balczarek-Strang K, Elsdon M, Dyson NJ: Functional antagonism between E2F family members. Gene Dev 2001, 15(16):2146-2160.

37. Wolf YI, Novichkov PS, Karev GP, Koonin EV, Lipman DJ: The universal distribution of evolutionary rates of genes and distinct characteristics of eukaryotic genes of different apparent ages. Proc Natl Acad Sci USA 2009, 106(18):7273-7280

38. Pal C, Papp B, Lercher MJ: An integrated view of protein evolution. Nat Rev Genet 2006, 7(5):337-348

39. Innan H, Kondrashov F: The evolution of gene duplications: classifying and distinguishing between models. Nat Rev Genet 2010, 11(2):97-108,

40. Zhang J: Evolution by gene duplication: an update. Trends in Ecology \& Evolution 2003, 18(6):292-298.

41. Sardet C, Vidal M, Cobrinik D, Geng Y, Onufryk C, Chen A, Weinberg RA: E2F-4 and E2F-5, two members of the E2F family, are expressed in the early phases of the cell cycle. Proc Natl Acad Sci USA 1995, , 92: 2403-2407.

42. Koreth J, van den Heuvel S: Cell-cycle control in Caenorhabditis elegans: how the worm moves from G1 to S. Oncogene 2001, 24(17):2756-2764.

43. Boxem M, van den Heuvel S: lin-35 Rb and cki-1 Cip/Kip cooperate in developmental regulation of G1 progression in C-elegans. Development 2001, 128(21):4349-4359.

44. Wildwater M, Campilho A, Perez-Perez JM, Heidstra R, Blilou I, Korthout $H$, Chatterjee J, Mariconti L, Gruissem W, Scheres B: The RETINOBLASTOMA- 
RELATED Gene Regulates Stem Cell Maintenance in Arabidopsis Roots. Cell 2005, 123(7):1337-1349.

45. Korenjak M, Taylor-Harding B, Binne UK, Satterlee JS, Stevaux O, Aasland R, White-Cooper H, Dyson N, Brehm A: Native E2F/RBF complexes contain Myb-interacting proteins and repress transcription of developmentally controlled E2F target genes. Cell 2004, 119(2):181-193.

46. Lewis PW, Beall EL, Fleischer TC, Georlette D, Link AJ, Botchan MR: Identification of a Drosophila Myb-E2F2/RBF transcriptional repressor complex. Gene Dev 2004, 18(23):2929-2940.

47. Harrison MM, Ceol CJ, Lu XW, Horvitz HR: Some C. elegans class B synthetic multivulva proteins encode a conserved LIN-35 Rb-containing complex distinct from a NuRD-like complex. Proc Natl Acad Sci USA 2006, 103(45):16782-16787.

48. Litovchick L, Sadasivam S, Florens L, Zhu XP, Swanson SK, Velmurugan S, Chen RS, Washburn MP, Liu XS, DeCaprio JA: Evolutionarily conserved multisubunit RBL2/p130 and E2F4 protein complex represses human cell cycle-dependent genes in quiescence. Molecular Cell 2007, 26(4):539-551.

49. Pilkinton M, Sandoval R, Colamonici OR: Mammalian Mip/LIN-9 interacts with either the p107, p130/E2F4 repressor complex or B-Myb in a cell cycle-phase-dependent context distinct from the Drosophila dREAM complex. Oncogene 2007, 26(54):7535-7543.

50. Schmit F, Korenjak M, Mannefeld M, Schmitt K, Franke C, von Eyss B, Gagrica S, Hanel F, Brehm A, Gaubatz S: LINC, a human complex that is related to $\mathrm{pRB}$-containing complexes in invertebrates regulates the expression of G(2)/M genes. Cell Cycle 2007, 6(15):1903-1913.

51. Desvignes T, Pontarotti P, Fauvel C, Bobe J: Nme protein family evolutionary history, a vertebrate perspective. BMC Evolutionary Biology 2009, 9(1):256.

doi:10.1186/1745-6150-5-55

Cite this article as: Cao et al:: The ancient function of RB-E2F Pathway: insights from its evolutionary history. Biology Direct 2010 5:55.

\section{Submit your next manuscript to BioMed Central and take full advantage of:}

- Convenient online submission

- Thorough peer review

- No space constraints or color figure charges

- Immediate publication on acceptance

- Inclusion in PubMed, CAS, Scopus and Google Scholar

- Research which is freely available for redistribution

Submit your manuscript at www.biomedcentral.com/submit 